\title{
MULTIPOLE ERROR ANALYSIS USING LOCAL 3-BUMP ORBIT DATA IN FERMILAB RECYCLER ${ }^{\dagger}$
}

\author{
M.J. Yang ${ }^{\ddagger}$, M. Xiao, Fermilab, Batavia, IL 60510, USA
}

\begin{abstract}
The magnetic harmonic errors of the Fermilab Recycler ring were examined using circulating beam data taken with closed local orbit bumps. Data was first parsed into harmonic orbits of first, second, and third order. Each of which was analyzed for sources of magnetic errors of corresponding order. This study was made possible only with the incredible resolution of a new BPM system that was commissioned after June of 2003.
\end{abstract}

\section{INTRODUCTION}

The Recycler Ring at Fermilab was built with all permanent magnets. The magnetic properties of all magnets used were measured before installation and surveyed in place to minimize possible errors. In spite of all efforts, it was soon realized that substantial amount of transverse plane coupling exists. After the commissioning of the new Recycler BPM system an analysis procedure, "Harmonic Orbit Decomposition" [1], was conceived. With this procedure it is possible to study magnetic errors one harmonic order at a time, without being confused by effects coming from other orders. The term "harmonic" is used synonymously to "multipole" within this write-up.

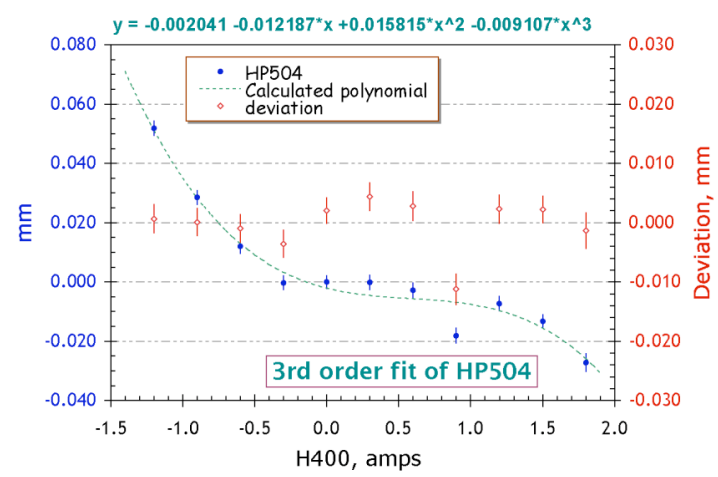

Figure 1. The position HP504 as a function of closed bump size, represented by the current on first corrector dipole $\mathrm{H} 400$. The fitted polynomial is shown in dotted line. The difference between data and the fitted polynomial is plotted in red with a different vertical scale, the vertical axis to the right.

Closed local orbit 3-bump was chosen as the mode of study. A 3-bump typically spans three consecutive locations in one plane, with the orbit oscillation starting at the first location and ending at the third location. With practically no orbit displacements anywhere around the ring, the effect of harmonic error vanishes, except within the 3-bump region.

So far the study has identified four locations as having substantial harmonic errors, RR620 and all three Lambertson magnet locations, i.e. 402, 328, and 214 locations. The summary of error fields found will be given at the

\footnotetext{
$\dagger$ Work supported by the US Department of Energy under contract DE-AC02-76CH00300.

\$ E-mail: YANG@FNAL.GOV
}

end. Detailed data analysis and calculation incorporating higher order errors will be shown only for 402 location.

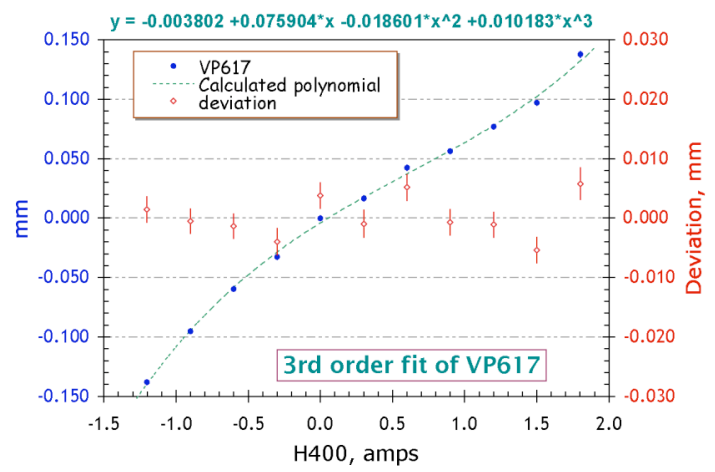

Figure 2. Example of vertical plane data of VP617 as a function of closed horizontal bump at 402. The effect of coupling is clear and non-linear.

\section{DATA}

At each location studied, orbit data was taken with magnitude of closed orbit bump varied in discrete steps. The step size was chosen such that at least eight data points were collected, see example in Figure 1. Circulating beam orbit is calculated by averaging 64 consecutive single turn position measurements. The corresponding RMS error is also recorded for statistical error propagation.

Two passes of data taking and analyses have been performed. In the first pass data was taken through out the ring in both horizontal and vertical plane, except where the 3-bump closure was known to be poor. Based on first pass analysis results [2], locations implicated as having substantial harmonic error were further studied in the second pass with greater detail and results are reported here.

\section{ANALYSIS}

\section{Harmonic orbits}

The first step of analysis is to apply the procedure of harmonic orbit decomposition and transform data set into orbits of different harmonic orders. Figure 1 shows one example of horizontal BPM data from HP504 with fitted polynomial. The deviation between data and fitted polynomial is plotted on a different vertical scale. Error bars are statistical errors of the position data. A second example, vertical BPM data from VP617, is shown in Figure 2. Note that no orbit motion is expected at HP504, which is far away from and outside of H402 3-bump, or VP617, which is in the other plane.

The derived harmonic orbits of first, second, and third order are shown in Figure 3 for horizontal plane and in Figure 4 for vertical plane. They are simply plots of coefficients of fitted polynomials from all BPMs in the Recycler Ring. The plot starts with downstream end of Proton injection Lambertson at 328 location, on the left, and ends with the Lambertson magnet itself on the right. 
The first order horizontal harmonic orbit, plot (a) of Figure 3, shows the 402 3-bump which starts at corrector dipole H400, peaks at 402 with about $8 \mathrm{~mm}$ in displacement, and closed by the corrector dipole at 404 location. The bump closure is clearly not perfect. Potential effects from harmonic errors elsewhere due to the ripple is expected to be small and out of place, and will be ignored.

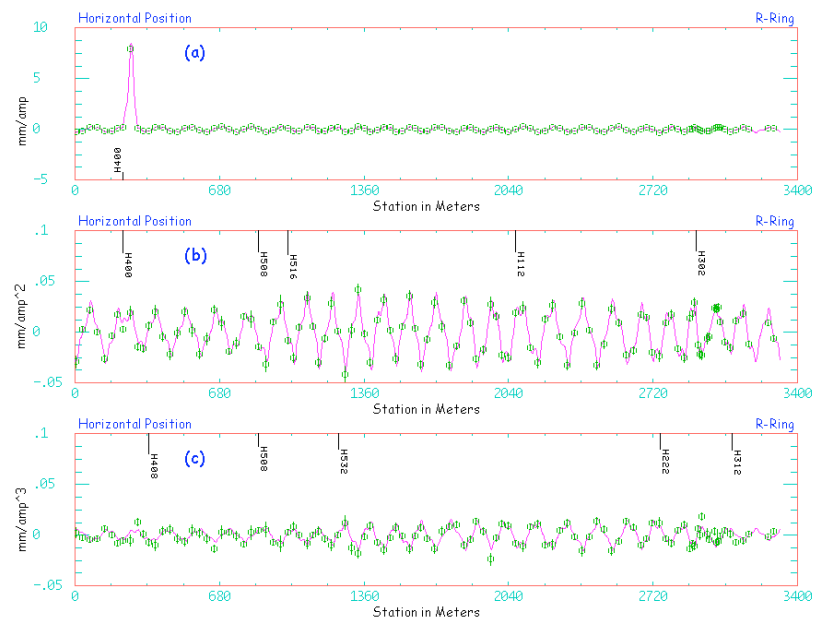

Figure 3. Ring-wide horizontal plane harmonic orbits from data taken with horizontal closed 3-bump at 402 location. From top are (a) first, (b) second, and (c) third order orbits. The magenta trace is the result of model calculation, with suspected error contributions.

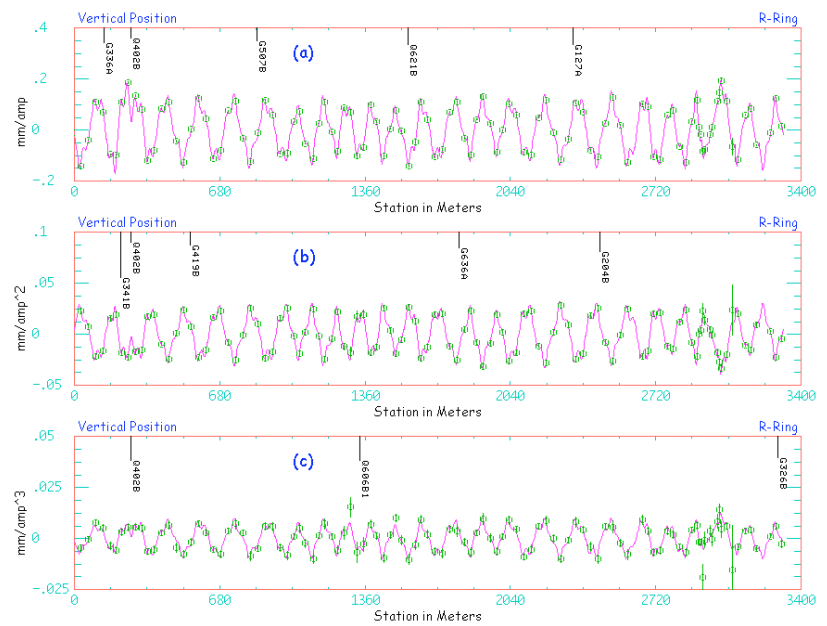

Figure 4. Ring wide vertical plane harmonic orbits from horizontal plane 402 closed 3-bump data. From top to bottom are (a) first, (b) second, and (c) third order orbits. In absence of skew harmonic error vertical orbits would be identically zero.

\section{Kick sources in the harmonic orbits}

The kick sources in the harmonic orbits are identified by MICADO/COCU [3] orbit fitting procedure and listed as text in Figure 5. They are included in the modeling calculation and plotted as the magenta traces in Figure 3 and 4, and their locations indicated by labels.

Listed from top to bottom in Figure 5 are fit results from MICADO/COCU for first, second, and third order harmonic orbits. Horizontal plane results on the left show correctors needed to smooth the horizontal orbit, their old setting, Amps equivalent of kicks required, and new set values. The first order result correctly identifies all three correctors used for the 3-bump as kick sources. Vertical plane results are listed on the right with names of quadrupoles and gradient magnets, the kicks needed to smooth the orbit, the equivalent displacements required in $\mathrm{mm}$, and in mils. Quadrupole and gradient magnet displacement were used to fit the vertical plane orbit mainly because 402 is a horizontal location where there is no vertical corrector dipole in the lattice.

Magnification factors of either 10 or 100 , as indicated with the fit results, were applied to the harmonic orbits before sending it to MICADO. This is done to maintain data precision since the inputs to MICADO procedure are in integer format and amplitudes of the harmonic orbits are very small, in actual physical scale.

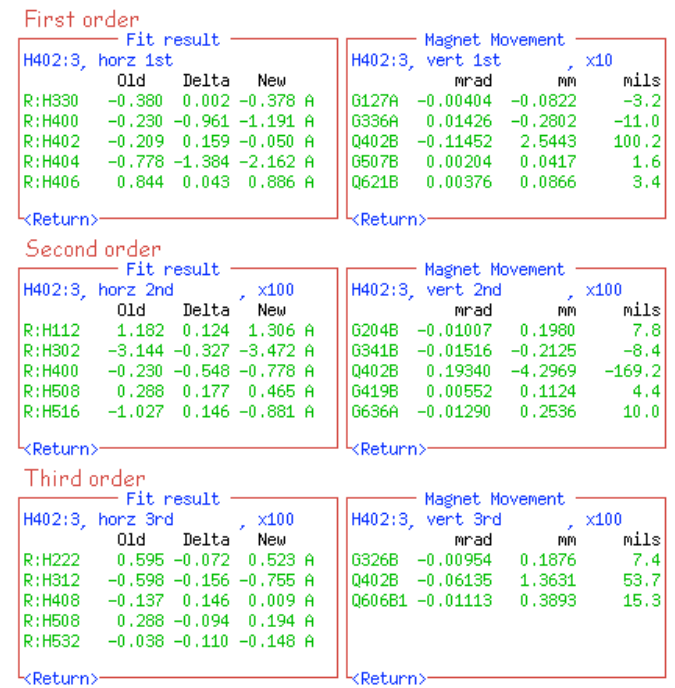

Figure 6. Result of using MICADO/COCU fitting procedure to find kick sources in the harmonic orbits from 402 horizontal closed bump data.

\section{Closed orbit stability}

In principle, kick errors are due to beam displacements at locations with substantial harmonic error. During analysis it was realized that corrector dipoles, while keeping the closed orbit centered to the aperture, were also moving it randomly on a 10- $\mu \mathrm{m}$ scale. In the plot of HP504 position against $\mathrm{H} 400$, Figure 1 , is one such data points at 0.9 amp. Random changes in closed orbit could introduce fictitious harmonic of any order and be a potential source of error, and more so for higher order harmonics. This problem can be seen on a global scale in plot (b) Figure 3, where the second order harmonic orbit amplitude changed several times. By using closed 3-bump for study it is safe to conclude that any kick error found outside the bump region would not be caused by harmonic errors.

Judging by the number of kicks needed to fit the orbit it is clear that only a few were drifting at any given time. The ability of MICADO to find kick error at 402 location was not compromised. As indicated in Figure 4 and listed in Figure 5 quadrupole Q402B was identified as source of kick error in all three order of vertical plane harmonic orbits. Same argument can be made for ignoring the effects due to imperfect bump closure, mentioned earlier. 
Table I Signature of magnetic harmonic errors.

\begin{tabular}{|l|l|l|}
\hline $\begin{array}{l}\text { Harmonic } \\
\text { components }\end{array}$ & $\begin{array}{l}\text { Bump } \\
\text { Horizontal }\end{array}$ & $\begin{array}{l}\text { Bump } \\
\text { Vertical }\end{array}$ \\
\hline quadrupole & closure & closure \\
\hline sextupole & closure & coupling \\
\hline octupole & closure & closure \\
\hline decapole & closure & coupling \\
\hline skew quadrupole & coupling & coupling \\
\hline skew sextupole & coupling & closure \\
\hline skew octupole & coupling & coupling \\
\hline skew decapole & coupling & closure \\
\hline
\end{tabular}

\section{Estimating strengths of multipole errors}

Depending on the primary displacement plane and order the effect of harmonic error alternates between causing bump closure distortion and cross-plane coupling. A quick reference for the signature of harmonic error is shown in Table I.

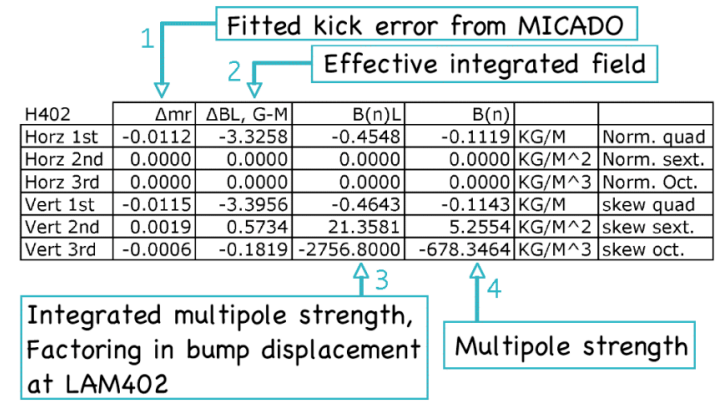

Figure 6. Steps in estimating strength of harmonic error for the 402 Lambertson magnet location.

Four steps in the harmonic analysis are shown in Figure 6 . The first has already been mentioned. In step 2 kick angles are converted to the equivalent integrated dipole field strength. The conversion to integrated multipole strengths in step 3 requires that error location be identified and horizontal bump displacement calculated. In this case LAM402 was assumed the culprit and, based on measured orbit, the corresponding horizontal displacement is interpolated using the modeling program. A geometric factor is then calculated for each harmonic order. In step 4 integrated strength is divided by the length of LAM402 magnet to get its multipole strength.

As shown in Figure 6 only first order error was found in the horizontal plane. For the vertical plane, errors were found in every order, implying the existence of skew quadrupole, skew sextupole, and skew octupole.

\begin{tabular}{|l|r|r|r|r|}
\cline { 2 - 5 } \multicolumn{1}{c|}{} & LAM402 & LAM328 & LAM214 & RR620 \\
\hline Quad, KG & -0.455 & -0.402 & -0.066 & 0.061 \\
\hline Sextupole, KG/M & 0.000 & 0.000 & 0.000 & 0.000 \\
\hline Octupole, KG/M^2 & 0.000 & 0.000 & 3222.516 & 0.000 \\
\hline Skew quad, KG & -0.464 & 0.086 & 0.172 & -0.486 \\
\hline Skew sextupole, KG/M & 21.358 & 20.141 & 31.434 & -3.361 \\
\hline Skew octupole, KG/M^2 & -2756.800 & 2898.115 & 2362.491 & 0.000 \\
\hline
\end{tabular}

Figure 7. Integrated multi-pole field strength as estimated from the analysis of the four locations studied.

\section{RESULTS}

Four locations have been studied in detail and listed in Figure 7. Of them, three are Lambertson locations, i.e. LAM402 for proton abort, LAM328 for anti-proton extraction, and LAM214 for anti-proton injection. The Lambertson magnets are prime suspects due in part to known production issues and to the fact that strong multipole errors had been observed in field region of LAM328. The fourth location, RR620, is within a short straight with no likely suspect. A rolled quadrupole is presumed.

Figure 8 plots results listed in Figure 7 in standard Fermilab units, used by Magnet Testing Facility (MTF), that is defined as $\left(L \cdot B_{n} / n !\right) \times 0.0254^{n} /\left(L_{\text {Bend }} \cdot B_{y} \cdot 10^{-4}\right)$. The conversion normalizes integrated multipole fields at $1 "$ to a unit that is equal to $6.182 \mathrm{KG}-\mathrm{M}$, the integrated dipole bend field, times $10^{-4}$. In this unit strengths of harmonic components are more comparable numerically. The order $n$ is 1 for quadrupole, 2 for sextupole, and 3 for octupole. The measured skew quadrupole component, averaged among arc magnets at defocusing locations, is $-1.0 \pm 1.2$ units, and the skew octupole component is $0.3 \pm 0.4$ units, well below the 10 units estimated from this analysis.

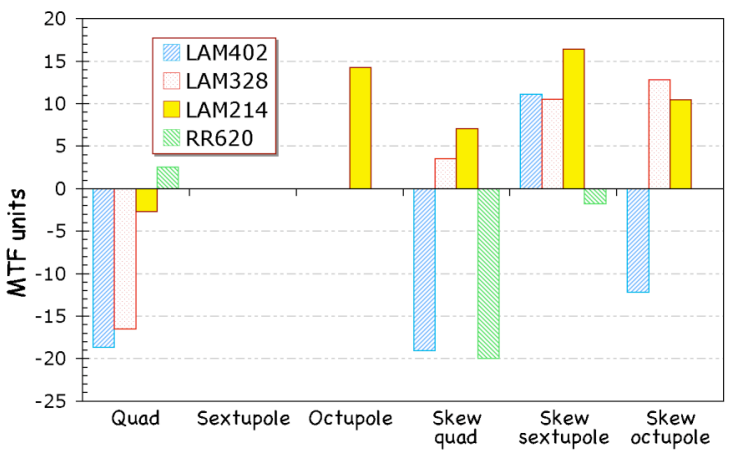

Figure 8. Multipole strength in normalized MTF unit for the four locations with detailed studies.

\section{CONCLUSION}

The Fermilab Recycler Ring magnetic errors were examined. The results reported. One location, RR620, has been confirmed to have a rolled quadrupole that is consistent with the analysis result. For the other three locations the analysis is based on the assumption that Lambertson magnets are the culprits. The fact that all three locations exhibit substantial harmonic errors in the field free region is itself very suggestive and could prove to be potentially serious. Further investigation is needed.

\section{ACKNOWLEDGEMENT}

The authors would like to thank D.E. Johnson for his valuable inputs during numerous discussions. Appreciation is also given to Instrumentation department for a fine Recycler Ring BPM system [4], which made the harmonic orbit study feasible.

\section{REFERENCES}

[1] M.J. Yang, "Harmonic Decomposition of Orbit Data for Multipole Analysis", this proceeding, MPPE083.

[2] M. Xiao et. al., "The Study on Coupling Issues in the Recycler at Fermilab", this proceeding.

[3] D. Brandt, W. Herr, J. Miles, and R. Schmidt, “A New Closed Orbit Correction Procedure for the CERN SPS and LEP”, Nucl. Inst. and Meth., A293(1990) 305

[4] Fermilab document RR-BPM-0001.rev1.1 